\title{
The Mexican National Programs on Teaching Mathematics and Science with Technology: The Legacy of a Decade of Experiences of Transformation of School Practices and Interactions
}

\author{
Ana Isabel Sacristán and Teresa Rojano \\ Center for Research and Advanced Studies (Cinvestav), Dept. of Mathematics Education, \\ Av. Instituto Politécnico Nacional 6058, D.F., Mexico \\ asacrist@cinvestav.mx, trojano@cinvestav.mx
}

\begin{abstract}
Here we give an overview of the Mexican experience of a national program, begun in 1997, of gradual implementation of computational tools in the lower secondary-school classrooms (children 12-15 years-old) for mathematics and science. This project illustrates, through the benefit of long-term hindsight, the successes and difficulties of large-scale massive implementation of technologies in schools. The key factors for success and for transforming school practices seem to be: adequate planning, gradual implementation, continuous training and support, and enough time (years) for assimilation and integration.
\end{abstract}

Keywords: Computers and technology in schools, national program, policymaking, teaching, mathematics, physics and science.

\section{A Government Initiative for the Use of Computational Technologies in Mexican Classrooms}

In 1997, the Mexican Ministry of Education (SEP) - in collaboration with several institutions such as the Latin-American Institute of Educational Communication (ILCE), the Center of Research and Advanced Studies (Cinvestav) and the National Autonomous University of Mexico (UNAM) - had the initiative to incorporate computational technologies to the primary and secondary (middle-school) levels. The aim was to 1) introduce in a gradual and systematic way the use of digital technologies into schools; 2) put into practice a meaningful use of those technologies using a pedagogical model that would improve and enrich curricular content; and 3) explore the use of technology for teaching to go beyond the curriculum and give early access to powerful ideas.

For secondary schools (children aged 12 to 15 years old) the initiative began with two parallel programs: "Teaching Mathematics with Technology" (EMAT) and "Teaching Physics with Technology" (EFIT). In a later phase, the project "Teaching Science through Mathematical Modeling" (ECAMM) was added, and EFIT evolved 
into the broader "Teaching Science with Technology" (ECIT) program to include Physics, Chemistry and Biology. (See [1] for a more comprehensive history of these programs).

These programs aimed to promote the use of new technologies, using a constructivist approach, to enrich and improve the current teaching and learning of mathematics and science in Mexico. A study [2] carried out in Mexico and England involving mathematical practices in science classes, revealed that few students were able to close the gap between the formal treatment of the curricular topics and their possible applications. This suggested that it was necessary to replace the formal approach of the then official curriculum in Mexico, with a "down-up" approach capable of fostering the students' explorative, manipulative, and communication skills.

Thus, a major part of these programs was to design activities and a pedagogical model for incorporating the use of technological tools to teaching that emphasized exploratory and collaborative learning. Technology and the computational instruments [3] are conceived as support tools mediating action [4] and students' construction of concepts. The principles characterizing the programs are summarized in the official documents (e.g. [5]) in the following way:

- A use of computer software or technological tools (e.g. calculators) that makes it possible to deal with concepts in a phenomenological way; provides representations of mathematical objects that can be directly manipulated; is related with a specific area of school knowledge content; e.g. for school mathematics: arithmetic, algebra, geometry, probability, etc.

- Specializes the users of the technology (teachers and students) in one or more pieces of software and/or tools so they become proficient in its use and are able to apply it for the teaching and learning of specific curricular topics.

- Puts into practice a collaborative model of learning: students work in pairs with one computer, thus promoting discussions and the exchange of ideas.

- Incorporates a pedagogical model where the teacher's role is that of promoting the exchange of ideas and collective discussion; at the same time, acting as mediator between the students and the technological tools (the computational environment), aiding the students in their work with the class activities and sharing with them the same expressive medium (tool).

The design of the pedagogical model, the choice of tools, and the activities was carefully carried out by researchers, both Mexican and international from top institutions in the world (who served as external international experts and advisors), taking into account results from studies in computer-based education for the practice in the "real world". For the pedagogical model, much of the philosophy and pedagogy underlying the design of mathematical microworlds [7] - which takes into account, not only the technical component, but the learner, the pedagogical and teacher components, as well as the contextual and social setting - was present in the design and recommendations for the EMAT and ECIT laboratories. Thus, emphasis was put on the changes in the classroom structure, such as the requirement of a different teaching approach and the way the classroom needs to be set up: from the physical set-up of the equipment, to the collaboration between students, to the role of the teacher, to the pedagogical tools (e.g. worksheets) [5]. In particular, the pedagogical model emphasizes a collaborative model of learning, with students working in pairs or teams for 
each computer (and the classroom computers set-up in a horseshoe fashion) for promoting discussions and the exchange of ideas.

The teacher's role is that of acting as a mediator between the students and the technological tools (the computational environment); guiding and aiding the students in their work with the class activities; intervening as necessary to ensure higher levels of conceptualization by students; promoting in students exploration, the formulation and validation of conjectures, the learning and analysis of mistakes, as well as the expression, exchange and collective debate of their ideas; and integrating the knowledge that is generated in the computational context with the more traditional mathematical knowledge.

The activities are organized through worksheets that aim to lead students to reflect on the work carried out with the technology and to synthesize it so that they can communicate it. The activities (piloted for over 3 years) of EMAT / ECIT are laid out in 16 books (most of them available for download at www.efit-emat.dgme.sep. gob.mx) that give a complete curricular development. The worksheets are intended to promote the model of collaborative work in the classroom. The sequences of activities were designed taking into account evolving lines in the different curriculum contents. For instance, for the mathematical activities: from arithmetic to algebra; from intuitive to exploratory dynamic geometry; from static descriptions to variation models; from solving closed problems to modeling.

Also, these worksheets, when filled-out, can give teachers information on students' work and the understandings of the concepts involved in a task.

EMAT and ECIT are designed to be carried out in a normal computer room, with a preference for software that allows open content (often shareware or freeware as well as software that is generally available in most computers - such as spreadsheets). ECIT also includes the use of simulators and sensors as well as interactive units for specific areas of the curriculum.

The gradual implementation of the EMAT and EFIT programs: From 1997-2000, the EMAT and EFIT programs were piloted in 14 states (out of 33) of Mexico, in 28 junior secondary schools. In the first year there were 33 participating teachers, which increased to 157 in the second year, and 905 in the third year. In this trial, we had the participation of national experts from the Center of Research and Advanced Studies (Cinvestav), the National Autonomous University of Mexico (UNAM), the Autonomous Technical Institute of Mexico (ITAM), and many state universities such as those of Coahuila, Durango, Colima, Morelos, San Luis Potosí, Aguascalientes, Michoacán e Hidalgo.

The programs were conceived to be expanded gradually in different ways, while preserving the quality of teacher training and of practice and implementation of the models in the classrooms. The ways of expansion were: 1) in the number of participating schools, teachers and students; 2) in regional coverage; 3) in the tools being used; 4) in curricular topics; 5) in school levels; and 6) in secondary school modalities (e.g. regular schools, technical schools, "tele-secondary" schools).

The expansion phase started in 2001. Adjustments were made that were derived from the results of the pilot phase, including adaptation of the tools being used. Implementation of the EMAT and ECIT programs expanded gradually and exponentially in the national public school system. Although we had conceived a gradual implementation everywhere, some states (such as Coahuila) decided to implement these models 
massively, and in those cases the support of local academic groups in universities was fundamental.

In terms of curricular expansion, the ECAMM (Teaching Science - Physics, Biology and Chemistry - through Mathematical Modeling) was developed as an extension to both EMAT and EFIT (although it follows naturally from EMAT), which uses mainly spreadsheet, graphing calculator and paper-and-pencil activities. Later, as mentioned above, EFIT was extended to include activities and materials for Chemistry and Biology, as well as Physics, becoming the ECIT (Teaching Science with Technology) program. Another extension was an attempt to adapt the models to the "Tele-secondary"1 (Telesecundaria) school model.

In terms of teacher-training, during the pilot stages, teacher-training was undertaken directly by the national and international experts, and there was continuous support of the teachers. In the expansion phase, however, due to the immensity of the scale of the program, this was no longer possible. A cascading model was implemented: the experts trained trainers who in turn trained teachers and/or head-teachers, who in turn were supposed to support other teachers. This carried with it the problem of a "faulty line effect" where the quality of the training was diluted (particular in that which concerns the understanding of the pedagogical model); but this was an unavoidable problem. In this sense we observed that the support of local academic groups (e.g. from local universities) or of a local advisor was crucial: having a local structure for needed support and continuous training has been observed to make a huge difference on whether teachers and/or schools continue to use the models or not.

In 2007, a change in government meant that federal support for the EMAT, EFIT, ECAMM and ECIT programs was dropped, but these programs continue in many states supported by the local state governments; and in other parts of the country more and more teachers use the program materials often simply because they find them useful.

\section{The EMAT Program}

One of the most extensively developed and implemented educational technology programs in Mexico has been the EMAT program. It followed the principles outlined in sections above, including the pedagogical model. In terms of the software and tools used in the EMAT program, a main criterion for their choice was to be the open tools [5]; that is where the user can be in control and has the power of deciding how to use the software. This allows for the construction of learning environments where students are able to decide on how to proceed, as opposed to other types of computer software that direct the student and the activity. These open tools have to be flexible enough so that they can be used with different didactical objectives, such as those designed for the program.

\footnotetext{
${ }^{1}$ The Telesecundaria School program - which began four decades ago, in the late sixties- is an educational model of the Ministry of Education for reaching the wider community (e.g. rural areas) that may not have access to regular lower secondary schools: in a Telesecundaria school, learning is mediated by a teacher-promoter for all subject areas, structured through learning guides, content guides, and television programs.
} 
In its first phase (1997-2000), using a pilot group of approximately 99 teachers and 10526 students in 8 states, the project researched the use of spreadsheets, CabriGéomètre, SimCalc, Stella and the TI-92 calculator, all aiming at covering curricular topics such as arithmetic, pre-algebra, algebra, geometry, variation and modeling. In the first year, different technology was implemented in different sites; the first generation groups used different technological tools, except for the calculator that was used by all groups. Specifically, spreadsheets were used in 1st Grade (children 12-13 yrsold); Cabri-Géomètre in second grade; and SimCalc in third grade; and the TI graphing calculator in all grades. In the following two years, the expansion was done gradually, by working with more teachers and grades in each school, thus giving the necessary training for each added tool. In this way, in the first three years, the participating teachers in that phase received training in at least three tools.

The conclusions derived from the experiences in this pilot phase were that it was easier for teachers to incorporate spreadsheets, the graphing calculator, and the dynamic geometry software into their practice. Thus, in the following expansion phase (2001-2006), these were the tools that were used, together with two added tools: Logo, and CAS (Computer Algebra System) activities with the calculator. The decision to add the Logo programming language was taken at the suggestion of both national and international advisors who evaluated the first phase and pointed out that there was still the need for more expressive tools and activities (such as programming), on the part of the students, and they suggested Logo. Logo had actually been considered since the beginning, but due to political factors, could not be included in the first phase. The addition of Logo proved to be very enriching in many schools, where often it became a students' favorite, but also enhanced and complemented the use of the other tools [6]. SimCalc and Stella were dropped because it was hard to fit these tools into the curriculum without a more extensive teacher-training that was hard to achieve. However, we are now reconsidering use of SimCalc, since the new curriculum implemented in 2007 includes elements of the mathematics of change and variation.

By the year 2003, the EMAT project had been implemented in 731 schools in 17 states, with 2283 participating teachers and close to 200,000 students (out of a population of over 5.7 million lower secondary school registered students); after that, many more states joined the program and teacher-training workshops were continuously held all over the country.

\section{The EFIT/ECIT Programs}

EFIT is the result of an adaptation to the Mexican school system of physics education, of the Canadian model: Technology Enhanced Science Secondary Instruction (TESSI). In EFIT, through activity guides, students explore physics concepts in four ways 1) through computer simulations; 2) through laboratory activities using sensors to collect data that is transferred to the computer; 3) carrying out experiments with computer software or multimedia equipment and 4) carrying out traditional physics laboratory experiments. These four approaches naturally give rise to "working stations" within a class session. 
The main tools initially used in EFIT, besides traditional physics laboratory equipment, were: Interactive Physics, for simulating real-world situations and visualizing phenomena; sensors for measuring real phenomena (originally the Pasco Probes: Pasco Introductory Physics Bundle and Smart Pulley); Nih Image: for processing and analyzing images; Internet; multimedia and videos; MSOffice; as well as an NCS Optical Scanner, and the LXR Test V. 51, Scoring edition and Interactive extension for computer-aided assessment.

In the expansion phase, the EFIT program began using mainly the Interactive Physics model and a set of sensors (which were, in later years, custom-made for the program) because these are enough to cover the curricular content. In that phase new physics activities were also designed, as well as expanding the model to include Chemistry and Biology activities, thus giving rise to ECIT.

\section{Results of the Evaluation and Assessment of the "Teaching with Technology" Programs}

Over the past decade, various studies have attempted to evaluate the projects from different perspectives. We know that from a theoretical point of view, the complexities of evaluating innovative computational environments - especially when they aim to be systemic - are far from resolved, and so this is a difficult task. Research has been carried out using both global and local levels of assessments. The global level focuses on understanding the educational system as a complex model that includes teachers, headmasters, authorities and parents as essential elements, whose observations also form part of the assessment of the project's collective ways of thinking about itself [8]. On the other hand, the local level concentrates on the specific learning of students, and the use of the tools with respect to student profiles combining both quantitative and qualitative (e.g. longitudinal case studies) research methodologies ${ }^{2}$. On the local level, we have been trying to gather data from two different angles, each of which informs the other.

On the one hand, we are assessing the use and implementation of the project tools, materials and pedagogical model. For this we use a variety of quantitative and qualitative instruments: field observations, interviews and questionnaires both for teachers and students. On the other hand, we need to evaluate students' learning. Since this is such a large-scale study, the evaluation of student learning has relied mainly on traditional school-mathematics items and quantitative techniques (e.g. pre- and post-tests; academic scores). Thus, not surprisingly, many results related to students' learning have been inconsistent. But data from the local level of assessment (written questionnaires and pupil interviews) from samples of students in selected states, was also used to analyze the evolution of skills and specific knowledge within the mathematics curriculum, as is reported in [9].

In general, research has shown that the programs have had a positive impact on students. For EMAT, it has been found that, in general, the use of the computational tools has had a very strong positive impact on children's attitudes towards mathematics, although the impact on learning is more difficult to assess. For example, [10]

\footnotetext{
${ }^{2}$ This research was sponsored, in part, by the CONACYT research grants G26338-S (until 2003) and 44632-S; in part by ILCE; as well as by other sources and grants.
} 
reports that there is a clear increase in students' enthusiasm and motivation; and although the impact is different for girls and for boys, the behavioral changes observed seem to lead to more gender equity.

The pilot phase (1997-2000), despite some difficulties, was groundbreaking in changing the role of the teacher and children: the changes in classroom dynamics modified the traditionally passive attitudes of children and empowered them, giving them a status almost equal (and sometimes even higher than the teacher) when involved in mathematical explorations with the tools [11]. The pilot phase thus created an irreversible change that seemed to indicate at the time, that it would allow for technologies to be incorporated, in an adequate way, into the Mexican school culture.

Despite these positive results, many issues and difficulties were detected. One finding is that the use of the technology made teachers aware of their deficiencies of their content knowledge, which had two types of consequences: in some cases this put teachers off wanting to work with technology; in other cases it motivated and helped teachers improve their content knowledge. We have also found that the teacher's attitudes and abilities towards the use of the technological tools and programs, have an impact on students' learning with the tools. Putting it bluntly, "good teachers" achieve good results: they are able to take advantage of the technological tools and their students benefit from those experiences; but less experienced, poorly trained teachers, or simply teachers who dislike the technological tools, do not do so well [9].

Other outstanding issues have been: lack of experience working with technology by both teachers and students; lack of adequate mathematical and content knowledge competency on the part of the teachers; difficulties in adapting to the proposed pedagogical model; teachers' lack of free time to prepare anything outside the established curriculum $^{3}$; lack of adequate follow-up teacher training and support because of administrative issues; many other bureaucratic difficulties; and lack of communication between the different levels of authorities.

In relation to the first issue - the lack of experience working with technology -in the years since the programs were first put into practice, teachers and students are now, more and more, much more familiar with computers prior to working in the technology programs. This is a cultural change as computers become more prevalent in society. But we still find many teachers that have difficulties and lack technical ability and self-confidence in the use of both the computer and the tools.

As to the other issues, none of these have been fully resolved. What became apparent since that pilot phase is that factors not present in laboratory settings come into play, when implementing a project such as this one, "out in the real world". (See [1] for comprehensive reports on the results of the associated investigations to the technology programs).

\section{The Technology Programs Ten Years Later, and Their Legacy}

The EMAT program, as well as the ECIT program to a lesser degree, have been a model in our country (and even internationally) of massive educational innovation

\footnotetext{
${ }^{3}$ This had as consequence that the EMAT worksheets became a means to structure the activities, which in turn also became much more directed than originally planned.
} 
whose main principle is that of using computational environments as a means for students to develop knowledge in new ways while opening paths of communication and social interaction in the classroom where meaningful discussions can take place between students and the teacher and amongst students. A decade of implementation of this program in secondary schools (children 12-15 yrs. old) in Mexico has left a wealth of experiences and results, which have been reported over the years and which we will attempt to synthesize and draw upon here. The characteristics of EMAT, in terms of its basic design principles; its implementation methodology; the diverse and extensive participating groups (researchers, software developers, educational authorities, teachers and students); its international background; and its close link with educational research, have implied that the decade of results and experiences that are derived from that program have had an extensive influence that surpasses its local experimental implementation. One area, in particular, that was influenced by EMAT is the curricular reform of the secondary school mathematics programs in Mexico and which has been an explicit reference for the use of technology in the curriculum of other countries.

Sadly, as mentioned above, in 2007, federal support for the EMAT/ECIT programs was dropped. However, the programs continue to thrive, particularly in some states such as Hidalgo, Durango, Morelos and Michoacán, where the programs were taken up by state authorities and/or by local university academic groups. In these states the programs have expanded gradually, with high-quality teacher-training plans and support groups led by local experts and teachers with long-term experience in the implementation of the EMAT/ECIT programs in schools. Some states, for example Hidalgo, have set-up a support and development structure with regional coordinators and head-teachers, as well as academic experts, who meet monthly and have developed an integrated syllabus with recommendations for the use of all the different tools, as well as new activities, according to the different areas and requirements of the curriculum. We consider this not only an important phase in the incorporation and development of the programs (where local groups take the initiative to enrich and complement the programs), but also an indicator of how the programs are appreciated and begin to be integrated into the broader school culture.

In another form of expansion, the programs and models have spread in other states, through teachers telling other teachers, who more and more demand support. This makes the lack of federal support a pity. We cannot keep track of how many teachers and/or schools have used the programs in this way, but we regularly get some information about this. Moreover, there are schools where teachers have become real enthusiasts of the program, using the different tools in an integrated way in their own activities and long-term school-projects (e.g. [12]), even achieving recognition for their work in technology-in-education events and fairs

Having teachers, schools, and states begin making the models and tools their own, incorporating them into their practices in an unforced manner, and complementing them with new activities is the long-term legacy of the programs. We feel that programs such as the ones discussed in this paper take a long time to properly take roots and develop. It is only now that we see these programs being assimilated into local educational systems in many parts of the country. But this is why we feel compelled to tell this story of a successful implementation program, as an example for researchers and policy-makers that the changes that can be brought about by the use of 
technology in schools can only happen adequately, through well thought-out programs, over long-terms of time and with adequate support. Those, we believe, are key factors: planning, gradual implementation (so that adjustments can be made), enough time (e.g. before expecting results) and continuous support.

\section{References}

1. Rojano, T. (ed.): Enseñanza de la Física y las Matemáticas con Tecnología: Modelos de Transformación de las Prácticas y la Interacción Social en el Aula. [The Teaching of Physics and Mathematics with Technology: Models of Transformation of Classroom Practices and Social Interaction]. SEP, Mexico (2006)

2. Rojano, T., Sutherland, R., Ursini, S., Molyneux, S., Jinich, E.: Ways of Solving Algebra Problems: The Influence of School Culture. In: Puig, L., Gutierrez, A. (eds.) Proceedings PME 20, vol. 4, pp. 219-226. Universidad de Valencia, Valencia (1996)

3. Balacheff, N., Kaput, J.: Computer-based Learning Environments in Mathematics. In: Bishop, A.J., Clements, K., Keitel, C., Kilpatrick, J., Laborde, C. (eds.) International Handbook of Mathematics Education, pp. 469-501. Kluwer Academic Publishers, Dordrecht (1996)

4. Wertsch, J.: Voices of the Mind: A Sociocultural Approach of Mediated Action. Harvester, London (1991)

5. Ursini, S., Rojano, T.: Guía para Integrar los Talleres de Capacitación EMAT. [Guide for integrating the EMAT training workshops]. SEP-ILCE, Mexico (2000)

6. Ursini, S., Sacristán, A.I.: On the Role and Aim of Digital Technologies for Mathematical Learning: Experiences and Reflections Derived from the Implementation of Computational Technologies in Mexican Mathematics Classrooms. In: Hoyles, C., Lagrange, J.-B., Le Hung, S., Sinclair, N. (eds.) Proceedings 17th ICMI Study Conference, Technology Revisited. Hanoi University of Technology, Hanoi (2006)

7. Hoyles, C., Noss, R.: A Pedagogy for Mathematical Microworlds. Educational Studies in Mathematics 23, 31-57 (1992)

8. Moreno, L., Rojano, T., Bonilla, E., Perrusquía, E.: The Incorporation of New Technologies to School Culture: The Teaching of Mathematics in Secondary School. In: Hitt, F., Santos, M. (eds.) Proceedings PME-NA 21, ERIC Clearinghouse for Science, Mathematics, and Environmental Education, Columbus, OH, vol. 2, pp. 827-832 (1999)

9. Trigueros, M., Sacristán, A.I.: Teachers' Practice and Students' Learning in the Mexican Programme for Teaching Mathematics With Technology. Int. J. Continuing Engineering Education and Life-Long Learning 18(5/6), 678-697 (2008)

10. Ursini, S., Sánchez, G., Orendain, M., Butto, C.: El Uso de las Tecnologías en el Aula de Matemáticas: Diferencias de Género Desde la Perspectiva de los Docentes. [The Use of Technologies in Classroom Mathematics: Gender Differences from the Teachers' Perspective]. Enseñanza de las Ciencias 22 (3), pp. 409.424 (2004)

11. Sacristán, A.I., Ursini, S., Trigueros, M., Gil, N.: Computational Technologies in Mexican Classrooms: The Challenge of Changing a School Culture. In: Dagiene, V., Mittermeir, R. (eds.) Information Technologies at Schools. 2nd Int. Conf. 'Informatics in Secondary Schools: Evolution and perspectives' Selected Papers, pp. 95-110. Institute of Mathematics and Informatics, Vilnius (2006)

12. Jiménez-Molotla, J., Gutiérrez-Gómez, A., Sacristán, A.I.: Painless Trigonometry: A Toolcomplementary School Mathematics Project. In: Kalaš, I. (ed.) Proceedings $11^{\text {th }}$ EuroLogo Conference: 40 Years of Influence on Education. Comenius University, Bratislava (2007) 